\title{
Differential prognostic values of mRNA expression of CEACAM gene family members in nonsmall cell lung cancer
}

This article was published in the following Dove Press journal:

Current Biomarker Findings

25 November 2016

Number of times this article has been viewed

\author{
Haruhiko Nakamura ${ }^{1,2}$ \\ Hiroki Sakai' \\ Tomoyuki Miyazawa' \\ Toshiaki Somehara ${ }^{2}$ \\ Noboru Nakayama ${ }^{2}$ \\ Kiyonaga Fujii ${ }^{2}$ \\ Toshihide Nishimura ${ }^{2}$ \\ 'Department of Chest Surgery, \\ ${ }^{2}$ Department of Translational Medicine \\ Informatics, St Marianna University \\ School of Medicine, Kawasaki, \\ Kanagawa, Japan
}

Abstract: Serum carcinoembryonic antigen (CEA) is widely used as a representative marker of various malignant tumors. CEA-related cell adhesion molecules (CEACAMs), including CEACAM5, are encoded in the human genome by 12 independent genes and can be potential targets for future cancer treatments. In nonsmall cell lung cancer, serum CEA levels have been reported to predict patient survival. However, associations between mRNA expression of CEACAM gene family members in tumor tissues and patient prognosis remain unclear. To clarify this point, we used the Kaplan-Meier plotter global portal site, which collects the results of Affymetrix gene expression microarray analyses from the publicly accessible Gene Expression Omnibus database and combined it with survival data of patients. A total of 1,926 nonsmall cell lung cancer patients were identified from the Gene Expression Omnibus series, Cancer Biomedical Informatics Grid, and The Cancer Genome Atlas databases. We found statistically significant associations between mRNA expression of several CEACAMs and overall survival (OS) in patients with nonsmall cell lung cancer and lung adenocarcinoma $(n=720)$ but not squamous cell carcinoma $(\mathrm{n}=524)$. In adenocarcinoma, higher expression levels of CEACAM6 and $C E A C A M 8$ were significantly associated with better OS, whereas higher expression levels of CEACAM3, CEACAM4, CEACAM19, and CEACAM21 were associated with worse OS. Conflicting results among multiple probe sets for the same gene were found for CEACAM1, $C E A C A M 5$, and CEACAM7. The findings of this study indicated that CEACAMs play important roles in tumor progression and impact OS of patients with adenocarcinoma. As the impact on OS differed based on the gene family members or the probe set used, the individual CEACAMs seem to function through complicated mechanisms. Further studies are necessary to resolve the problems encountered in our present study.

Keywords: mRNA, microarray, survival, nonsmall cell lung cancer, CEACAM, CEA

\section{Introduction}

Approximately 50 years ago, carcinoembryonic antigen (CEA) was identified as an oncofetal antigen in colorectal cancer in addition to normal human fetal organs, including the gut, liver, and pancreas. ${ }^{1}$ Further studies revealed the presence of CEA and numerous CEA cross-reacting antigens in human sera, normal tissues, and various cancers other than colorectal cancer. ${ }^{2-5}$ A family of CEA-related cell adhesion molecules (CEACAMs), including CEACAM5, is known to be encoded in the human genome by 12 independent genes on chromosome 19q13., 2,6,7 These CEACAM proteins belong to the immunoglobulin supergene family, and the molecules contain one or two variable-like domains with or without constant 2 -like domains. ${ }^{2} \mathrm{CEA}$ is widely used as a representative serum tumor marker of various malignant tumors and elevated serum
Correspondence: Haruhiko Nakamura Department of Chest Surgery, St Marianna University School of Medicine, 2-16-I Sugao, Miyamae-ku, Kawasaki, Kanagawa 216-85II, Japan

Tel +8I 449778 III, ext. 3230

Fax +81449765792

Email h-nakamura@marianna-u.ac.jp 
CEA levels are reported to be frequently associated with a poor clinical outcome in cancer patients presumably through a variety of mechanisms, including the promotion of invasion, dissemination, metastasis, and immune suppression. ${ }^{2}$ Serum CEA concentrations are increased in both nonsmall cell lung cancer (NSCLC) and small cell lung cancer. ${ }^{8-10}$ In NSCLC, elevated serum CEA levels have been reported to be associated with histological types, advanced disease stages, and worse prognoses. ${ }^{8,11-15}$ More recently, vaccination therapy, antibody therapy, and small interfering RNA therapy targeting CEACAMs have been developed as new therapies for several solid tumors, including lung cancer. ${ }^{16-22}$ Thus, CEA and related molecules are important not only for diagnoses but also for future therapeutic targets in various malignant tumors. ${ }^{23}$

In spite of many reports regarding associations of serum CEA levels and prognosis of NSCLC, information about mRNA expression in tumor tissues and its relationship to patient survival is quite limited. Thus, we studied associations between mRNA expression detected by gene expression microarrays and overall survival (OS) in NSCLC patients by accessing an online public database. ${ }^{24,25}$ To the best of our knowledge, this is the first report focusing on associations between mRNA expression of the CEACAM gene family members and OS in NSCLC patients.

\section{Materials and methods}

Gene Expression Omnibus (GEO; www.ncbi.nlm.nih. gov/geo/) is a public functional genomics data repository supported by the National Center for Biotechnology Information (http://www.ncbi.nlm.nih.gov/). Based on the GEO database for NSCLC, the Kaplan-Meier plotter global portal site (http://kmplot.com/analysis/index. $\mathrm{php}$ ? $\mathrm{p}=$ service \&cancer=lung) provides combined data of mRNA expression of independent genes on gene expression microarrays and survival data of patients with NSCLC. ${ }^{24}$ Microarray platforms in the provided database were Affymetrix HG-U133A (GPL96), HG-U133 Plus 2.0 (GPL570), and HG-U133A 2.0 (GPL3921). These microarrays have 22,277 probe sets in common. ${ }^{24} \mathrm{~A}$ total of 1,926 NSCLC patients were identified from the GEO series, Cancer Biomedical Informatics Grid (https://github.com/ NCIP/caarray; caArray project), and The Cancer Genome Atlas (http://cancergenome.nih.gov) database. The data sets used in the present study are shown in Table $1 .^{26-37}$ Characteristics of the CEACAM gene family members are listed in Table 2., ${ }^{2,6,7}$ Associations between mRNA expression profiles of nine CEACAM genes (CEACAM1, CEACAM3,
Table I Datasets of nonsmall cell lung cancer included in the analysis

\begin{tabular}{|c|c|c|c|c|c|c|}
\hline Dataset & Platform & $\begin{array}{l}\text { Sample } \\
\text { size }\end{array}$ & M & $\mathbf{F}$ & $\begin{array}{l}\text { Stage } \\
\text { I/II/III/IV }\end{array}$ & $\begin{array}{l}\text { Histology } \\
\text { AD/SQ }\end{array}$ \\
\hline GSE4573 & GPL96 & 130 & 82 & 48 & $73 / 34 / 23 / 0$ & $0 / 130$ \\
\hline GSEI48I4 & GPL96 & 89 & 66 & 23 & $45 / 44 / 0 / 0$ & $27 / 52$ \\
\hline GSEI9188 & GPL570 & 83 & 59 & 24 & NA & $4 I / 24$ \\
\hline GSE3 I 4I & GPL570 & 111 & NA & NA & NA & $58 / 53$ \\
\hline GSE3 1210 & GPL570 & 226 & 105 & 121 & $168 / 58 / 0 / 0$ & $226 / 0$ \\
\hline caArray & GPL96 & 468 & 240 & 228 & NA & NA \\
\hline TCGA & GPL392I & 74 & 49 & 25 & NA & 0/7I \\
\hline GSE290I 3 & GPL570 & 55 & 38 & 17 & $24 / 14 / 17 / 0$ & $30 / 25$ \\
\hline GSE37745 & GPL570 & 196 & 107 & 89 & $130 / 35 / 27 / 4$ & $106 / 66$ \\
\hline GSE30219 & GPL570 & 293 & 252 & 41 & NA & $85 / 61$ \\
\hline GSE3। 908 & GPL96 & 20 & 4 & 16 & $10 / 5 / 3 / 0$ & $20 / 0$ \\
\hline GSE5008I & GPL570 & 181 & 98 & 83 & $127 / 54 / 0 / 0$ & $127 / 42$ \\
\hline Total & & 1,926 & 1,100 & 715 & $652 / 320 / 70 / 4$ & $720 / 524$ \\
\hline
\end{tabular}

Abbreviations: $A D$, adenocarcinoma; $F$, females; $M$, males; NA, not applicable; SQ, squamous cell carcinoma.

CEACAM4, CEACAM5, CEACAM6, CEACAM7, CEACAM8, $C E A C A M 19$, and $C E A C A M 21$ ) in tumor tissues and OS of NSCLC patients were studied. Among the $C E A C A M$ genes, three (CEACAM16, CEACAM18, and CEACAM20) were not included in the studied microarrays.

On the Affymetrix gene expression microarrays, six $C E A C A M$ genes were detected by multiple different probe sets: five probes for $C E A C A M 1$, three for $C E A C A M 3$ and $C E A C A M 7$, and two for CEACAM5, CEACAM6, and $C E A C A M 21$. A single reliable probe set matching each of the examined genes was determined by assessing specificity, splice isoform coverage, and robustness against transcript degradation. These selected probes are marked as Jetset probes $^{38}$ on the webpage.

The OS rates of two groups of patients subdivided by the median value of mRNA expression were calculated, and Kaplan-Meier survival plots were obtained from the webpage. Simultaneously, the hazard ratio of the higher expression group relative to the lower expression group, 95\% confidence intervals, and log-rank $p$-values were automatically calculated on the same webpage.

\section{Results}

Differences in OS between groups with higher and lower expression levels of the investigated CEACAM genes are shown according to histological types: NSCLC, adenocarcinoma (AD), and squamous cell carcinoma (SQ; Table 3). Expression of most $C E A C A M$ family members was significantly associated with OS in patients with NSCLC $(n=1,926)$. Similar results were obtained for patients with $\operatorname{AD}(n=720)$. In contrast, none of the examined $C E A C A M$ gene family members were associated with OS in patients with SQ $(n=524)$, suggesting 
Table 2 Full-length structure, number of splice variants, and function of the CEACAM family members

\begin{tabular}{|c|c|c|c|c|c|}
\hline $\begin{array}{l}\text { CEACAM family } \\
\text { members }\end{array}$ & N-domain & $\begin{array}{l}\text { C2-like Ig } \\
\text { domain }\end{array}$ & $\begin{array}{l}\text { Membrane } \\
\text { anchorage }\end{array}$ & $\begin{array}{l}\text { Splice } \\
\text { variants }^{\mathbf{a}}\end{array}$ & Known or speculated functions \\
\hline CEACAMI & 1 & 3 & Transmembrane & 13 & $\begin{array}{l}\text { CEACAMI-L/CEACAM-S ratio may be associated with } \\
\text { metastasis and shorter survival in several malignant tumors }\end{array}$ \\
\hline CEACAM3 & I & 0 & Transmembrane & 7 & Phagocytosis of specific bacterial pathogens \\
\hline CEACAM4 & 1 & 0 & Transmembrane & 2 & $\begin{array}{l}\text { Phagocytosis of specific bacterial pathogens, expressed in } \\
\text { medullary thyroid carcinoma cells }\end{array}$ \\
\hline CEACAM5 & 1 & 6 & GPI-linkage & 9 & $\begin{array}{l}\text { Connecting adjacent epithelial cell membranes in both } \\
\text { embryonic intestine and colon cancer, inhibition of } \\
\text { differentiation, suppression of tumor immunity }\end{array}$ \\
\hline CEACAM6 & 1 & 2 & GPI-linkage & 1 & $\begin{array}{l}\text { Tamoxifen resistance in breast cancer, inhibition of } \\
\text { differentiation in colon cancer }\end{array}$ \\
\hline CEACAM7 & 1 & I & GPI-linkage & 3 & $\begin{array}{l}\text { Downregulated in colon cancer, lower expression may be } \\
\text { predictive of rectal cancer recurrence }\end{array}$ \\
\hline CEACAM8 & 1 & 2 & GPI-linkage & 2 & Myelofibrotic transformation, released from human granulocyte \\
\hline CEACAMI6 & 2 & 2 & Free & 1 & Hearing in the inner ear \\
\hline CEACAMI 8 & 1 & 2 & Transmembrane & 0 & No information \\
\hline CEACAMI9 & I & 2 & Transmembrane & 8 & Progression of breast cancer \\
\hline CEACAM20 & I & 6 & Transmembrane & 5 & Downregulated in prostate cancer, tubule formation \\
\hline CEACAM 2 I & I & I & Transmembrane & 6 & Candidate gene for schizophrenia \\
\hline
\end{tabular}

Note: ${ }^{2}$ Number of variants were obtained from Ensemble (http://asia.ensembl.org/index.html).

Abbreviations: CEA, carcinoembryonic antigen; CEACAM, CEA-related cell adhesion molecule; Ig, immunoglobulin; GPI, glycosylphosphatidylinositol.

Table 3 Overall survival differences in patients with nonsmall cell lung cancer based on mRNA expression of the CEACAM gene family members

\begin{tabular}{|c|c|c|c|c|c|c|c|}
\hline \multirow{2}{*}{$\begin{array}{l}\text { CEACAM } \\
\text { family }\end{array}$} & \multirow{2}{*}{$\begin{array}{l}\text { Probes } \\
\text { Affymetrix ID }\end{array}$} & \multirow{2}{*}{$\begin{array}{l}\text { NSCLC }(n=I, 926) \\
\text { HR }(95 \% \mathrm{Cl}) \text { high } \\
\text { expression }\end{array}$} & \multirow[t]{2}{*}{$p$-Value } & \multirow{2}{*}{$\begin{array}{l}\text { AD }(n=720) \\
\text { HR }(95 \% \mathrm{CI}) \text { high } \\
\text { expression }\end{array}$} & \multirow[t]{2}{*}{$p$-Value } & \multirow{2}{*}{$\begin{array}{l}\text { SQ }(n=524) \\
\text { HR }(95 \% \mathrm{Cl}) \text { high } \\
\text { expression }\end{array}$} & \multirow[t]{2}{*}{$p$-Value } \\
\hline & & & & & & & \\
\hline \multirow[t]{5}{*}{ CEACAMI } & 209498_at ${ }^{\mathrm{a}}$ & $0.67(0.59-0.76)$ & $<0.0001$ & $0.78(0.62-0.98)$ & 0.034 & $0.94(0.74-1.19)$ & 0.61 \\
\hline & 206576_s_at & $0.99(0.87-I .12)$ & 0.83 & $1.35(1.07-1.7)$ & 0.011 & $0.95(0.75-1.2)$ & 0.66 \\
\hline & 210610_at & $0.84(0.74-0.95)$ & 0.007 & $0.76(0.6-0.97)$ & 0.024 & $0.94(0.74-1.19)$ & 0.58 \\
\hline & 211883_x_at & $0.92(0.82-1.05)$ & 0.23 & $1.09(0.87-1.38)$ & 0.44 & $0.97(0.76-1.22)$ & 0.78 \\
\hline & 211889_x_at & $0.92(0.82-1.05)$ & 0.23 & $1.27(1.01-1.6)$ & 0.042 & $0.95(0.75-1.2)$ & 0.65 \\
\hline \multirow[t]{3}{*}{ CEACAM3 } & 208052_x_at & $1.29(1.14-1.47)$ & $<0.0001$ & $1.66(1.32-2.1)$ & $<0.0001$ & $1.07(0.84-1.36)$ & 0.59 \\
\hline & 210789_x_at & $1.23(1.09-1.4)$ & 0.0012 & $1.46(1.16-1.84)$ & 0.0014 & $1.09(0.86-1.38)$ & 0.49 \\
\hline & 217209_at & I.I4 (I-I.29) & 0.047 & $1.54(1.22-1.95)$ & 0.00026 & $\mathrm{I} .03(0.8|-| .3 \mid)$ & 0.8 \\
\hline CEACAM4 & 207205 at $^{\mathrm{a}}$ & $1.24(1.09-1.4)$ & 0.001 & $1.65(1.31-2.09)$ & $<0.0001$ & $1.02(0.8-1.3)$ & 0.87 \\
\hline \multirow[t]{2}{*}{ CEACAM5 } & 201884_at ${ }^{\mathrm{a}}$ & $0.98(0.87-I . I I)$ & 0.77 & $1.04(0.82-\mid .31)$ & 0.77 & $0.88(0.69-1.1 \mathrm{I})$ & 0.28 \\
\hline & 217291_at & $1.27(1.12-1.44)$ & 0.00022 & $1.45(1.15-1.83)$ & 0.0019 & $1.21(0.96-1.54)$ & 0.11 \\
\hline \multirow[t]{2}{*}{ CEACAM6 } & 211657 at $^{\mathrm{a}}$ & $0.7(0.62-0.79)$ & $<0.0001$ & $0.68(0.54-0.85)$ & 0.00097 & $0.97(0.77-1.23$ & 0.83 \\
\hline & 203757_s_at & $0.67(0.59-0.77)$ & $<0.0001$ & $0.66(0.52-0.83)$ & 0.00039 & $1.06(0.83-1.34)$ & 0.66 \\
\hline \multirow[t]{3}{*}{ CEACAM7 } & 206198_s_at & I.I (0.97-I.25) & 0.14 & $1.33(1.06-1.68)$ & 0.015 & $0.93(0.74-1.18)$ & 0.57 \\
\hline & 206199_at ${ }^{\mathrm{a}}$ & $1.16(1.02-1.31)$ & 0.025 & $1.03(0.8 \mathrm{I}-1.3)$ & 0.81 & $1.06(0.84-1.35)$ & 0.62 \\
\hline & 211848_s_at & $0.99(0.87-1.12)$ & 0.84 & $1.12(0.89-1.4 I)$ & 0.34 & $0.92(0.73-1.17)$ & 0.52 \\
\hline CEACAM8 & 206676_at ${ }^{\mathrm{a}}$ & $0.77(0.67-0.87)$ & $<0.0001$ & $0.7 \mid(0.56-0.9)$ & 0.0038 & $0.82(0.65-1.04)$ & 0.11 \\
\hline CEACAMI9 & 230504_at ${ }^{\mathrm{a}}$ & $1.27(1.08-1.5)$ & 0.0043 & $1.6(1.25-2.05)$ & 0.00017 & $0.99(0.73-1.35)$ & 0.97 \\
\hline \multirow[t]{2}{*}{ CEACAM2I } & 214907 at $^{\mathrm{a}}$ & I.II (0.98-1.26) & 0.097 & $1.6(1.27-2.02)$ & $<0.0001$ & I (0.79-I.27) & 0.99 \\
\hline & 216605_at & $\mathrm{I} .07(0.94-\mid / 2 \mathrm{I})$ & 0.3 & I.4 (I.II-I.77) & 0.0047 & $0.97(0.77-1.23)$ & 0.81 \\
\hline
\end{tabular}

Notes: aletset probe. Statistically significant $p$-values are indicated in bold.

Abbreviations: AD, adenocarcinoma; CEACAM, carcinoembryonic antigen-related cell adhesion molecule; Cl, confidence interval; $\mathrm{HR}$, hazard ratio; NSCLC, nonsmall cell lung cancer; SQ, squamous cell carcinoma.

that the significant differences in OS among NSCLC patients were mainly due to OS differences in AD patients.

The prognostic value of mRNA expression of CEACAM genes in $\mathrm{AD}$ tissues according to the individual probe sets of the microarrays are summarized in Table 4. Higher expression of CEACAM6 and CEACAM8 was associated with better OS, whereas higher expression of CEACAM3, CEACAM4, CEACAM19, and CEACAM21 was associated with worse OS. Conflicting results among multiple probe sets for the same gene were found in CEACAM1, CEACAM5, and CEACAM7. 
Table 4 Prognostic significance of higher mRNA expression of the CEACAM gene family members in lung adenocarcinoma based on individual probes

\begin{tabular}{|c|c|c|c|}
\hline CEACAM family & Better OS & Worse OS & NS \\
\hline \multirow[t]{2}{*}{ CEACAMI } & 209498_at ${ }^{\mathrm{a}}$ & 206576_s_at & 211883_x at \\
\hline & 210610_at & 211889_x_at & \\
\hline \multirow[t]{3}{*}{ CEACAM3 } & & 208052_x_at & \\
\hline & & 210789_x_at & \\
\hline & & 217209_at & \\
\hline CEACAM4 & & 207205_at ${ }^{\mathrm{a}}$ & \\
\hline CEACAM5 & & 217291_at & $201884 \mathrm{at}^{\mathrm{a}}$ \\
\hline \multirow{2}{*}{ CEACAM 6} & $211657 \mathrm{at}^{\mathrm{a}}$ & & \\
\hline & 203757_s_at & & \\
\hline \multirow[t]{2}{*}{ CEACAM7 } & & 206198_s_at & 206199_at ${ }^{\mathrm{a}}$ \\
\hline & & & 211848_s_at \\
\hline CEACAM8 & 206676_at ${ }^{\mathrm{a}}$ & & \\
\hline CEACAM 19 & & 230504_at ${ }^{\mathrm{a}}$ & \\
\hline \multirow[t]{2}{*}{ CEACAM2I } & & 214907_at $\mathrm{at}^{\mathrm{a}}$ & \\
\hline & & 216605_at & \\
\hline
\end{tabular}

Note: ${ }^{\text {Jetset probe. }}$

Abbreviations: CEACAM, carcinoembryonic antigen-related cell adhesion molecule; OS, overall survival; NS, no significant difference in OS.

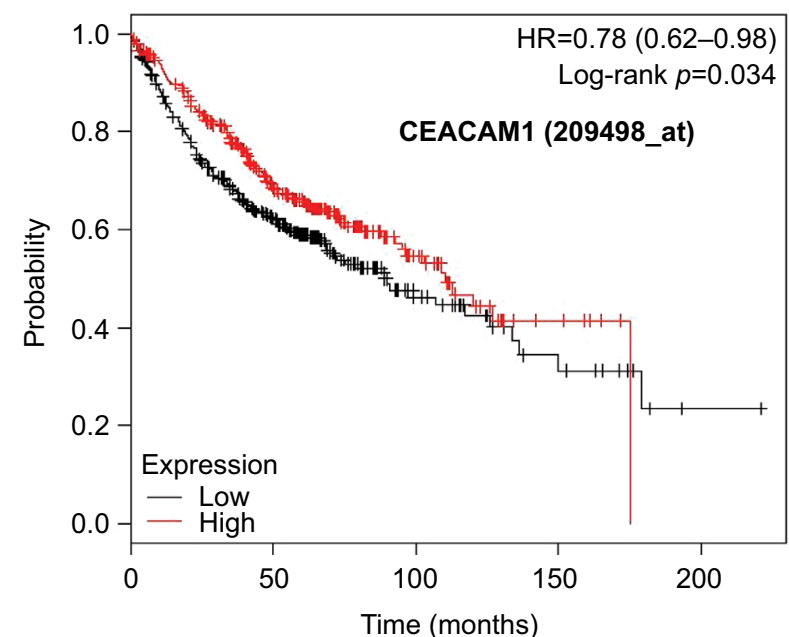

Number at risk

$\begin{array}{ccccc}\text { Low } 360 & 168 & 32 & 11 & 1 \\ \text { High } 360 & 180 & 37 & 8 & 0\end{array}$

Figure I Kaplan-Meier survival curves of the higher and lower expression groups divided by the median value of CEACAMI (Jetset probe, 209498_at) in patients with adenocarcinoma.

Note: Overall survival was better in the higher expression group $(p=0.034)$. Abbreviation: HR, hazard ratio.

Kaplan-Meier plots of OS using the Jetset probes for individual genes are shown in Figures 1-9. Because the online database listed no Jetset probe for CEACAM3, 208052_x_at was used as a representative probe for this gene to construct the OS curves.

\section{Discussion}

Quantification of serum CEA in lung cancer patients is widely performed to arrive at a diagnosis, evaluate tumor responses

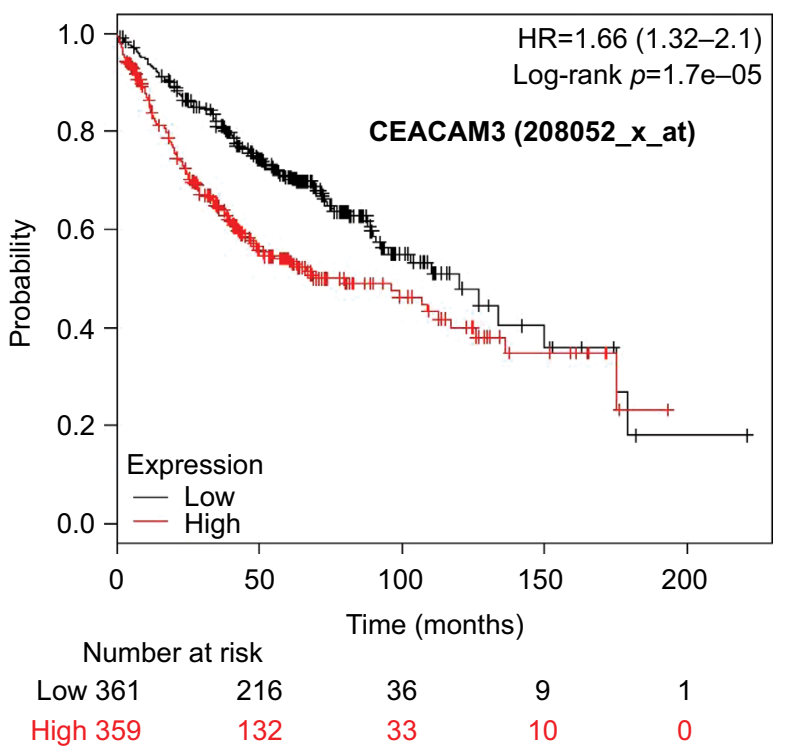

Figure 2 Kaplan-Meier survival curves of the higher and lower expression groups divided by the median value of CEACAM3 (probe, 208052_x_at) in patients with adenocarcinoma.

Note: Overall survival was worse in the higher expression group $(p<0.0001)$. Abbreviation: HR, hazard ratio.

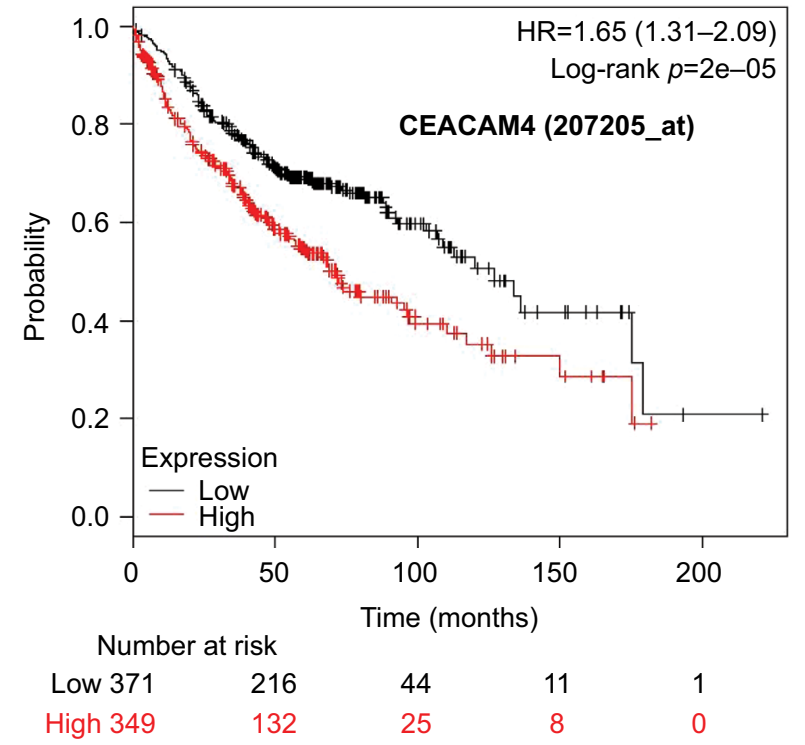

Figure 3 Kaplan-Meier survival curves of the higher and lower expression groups divided by the median value of CEACAM4 (Jetset probe, 207205_at) in patients with adenocarcinoma.

Note: Overall survival was worse in the higher expression group $(p<0.000$ I).

Abbreviation: $\mathrm{HR}$, hazard ratio.

to various therapeutic modalities, and predict risks of postsurgical recurrences. However, evidence of prognostic values in lung cancer patients remains unclear. Because of this, there are no official guidelines or recommendations for the use of CEA as a prognostic indicator of lung cancer.

According to a recent review article regarding the prognostic significance of CEA in lung cancer, 18 studies reported statistically significant evidence for the use of CEA as a 


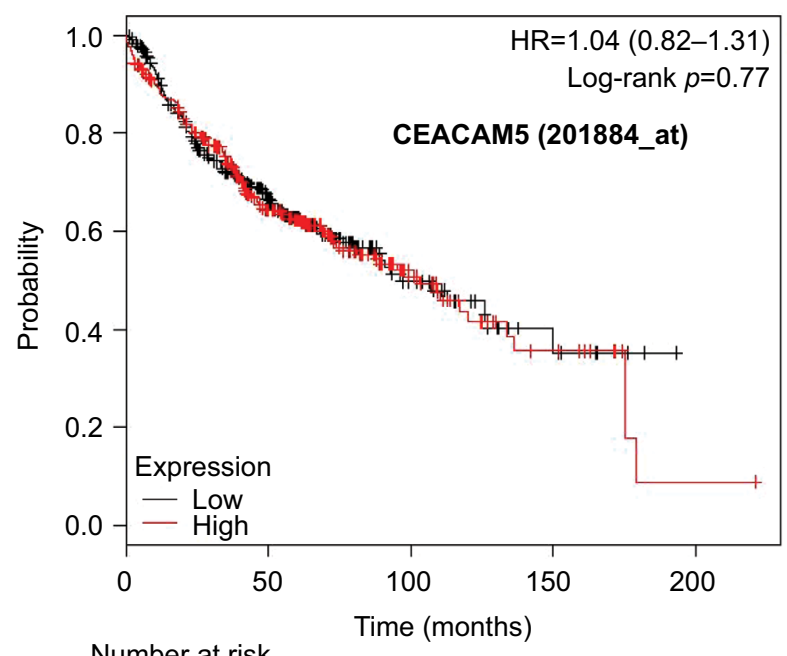

Number at risk

$\begin{array}{ccccc}\text { Low } 360 & 173 & 32 & 8 & 0 \\ \text { High } 360 & 175 & 37 & 11 & 1\end{array}$

Figure 4 Kaplan-Meier survival curves of the higher and lower expression groups divided by the median value of CEACAM5 (Jetset probe, 201884_at) in patients with adenocarcinoma.

Note: There was no significant difference in overall survival between the two groups $(p=0.77)$.

Abbreviation: $\mathrm{HR}$, hazard ratio.

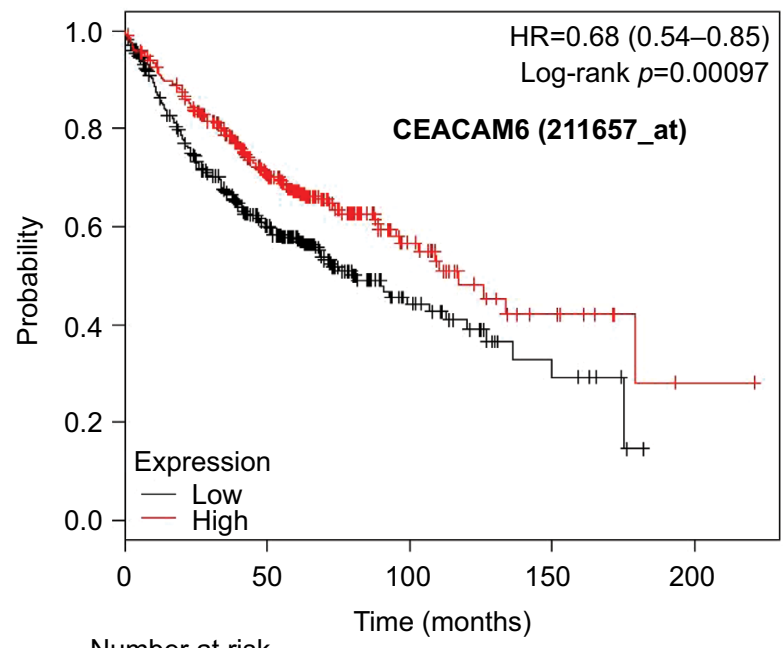

$\begin{array}{llccc}\text { Low } 360 & 160 & 33 & 9 & 0 \\ \text { High } 360 & 188 & 36 & 10 & 1\end{array}$

Figure 5 Kaplan-Meier survival curves of the higher and lower expression groups divided by the median value of CEACAM6 (Jetset probe, 211657_at) in patients with adenocarcinoma.

Note: Overall survival was better in the higher expression group $(p=0.00097)$. Abbreviation: $H R$, hazard ratio.

prognostic marker in NSCLC patients, while seven studies showed negative results. ${ }^{39}$ Among the 25 studies included in this review article, only one examined the relationship between immunohistochemical CEA expression in tumor tissues and prognoses of patients but found no association. ${ }^{40} \mathrm{~A}$ meta-analysis of 16 studies (4,296 NSCLC patients) reported that preoperative high serum CEA levels were associated

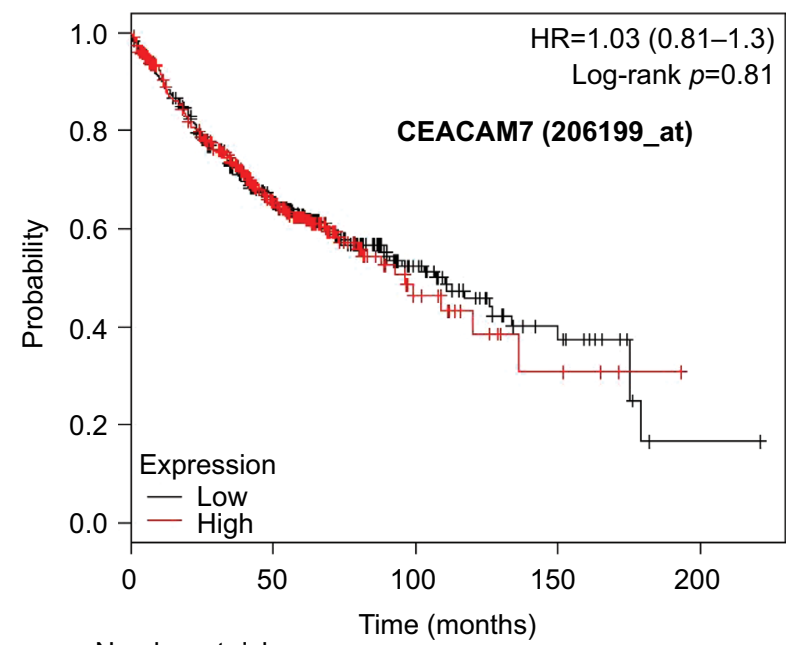

Number at risk

$\begin{array}{ccccc}\text { Low } 362 & 194 & 49 & 15 & 1 \\ \text { High } 358 & 154 & 20 & 4 & 0\end{array}$

Figure 6 Kaplan-Meier survival curves of the higher and lower expression groups divided by the median value of CEACAM7 (Jetset probe, 206199_at) in patients with adenocarcinoma.

Note: There was no significant difference in overall survival between the two groups $(p=0.81)$.

Abbreviation: $H R$, hazard ratio.

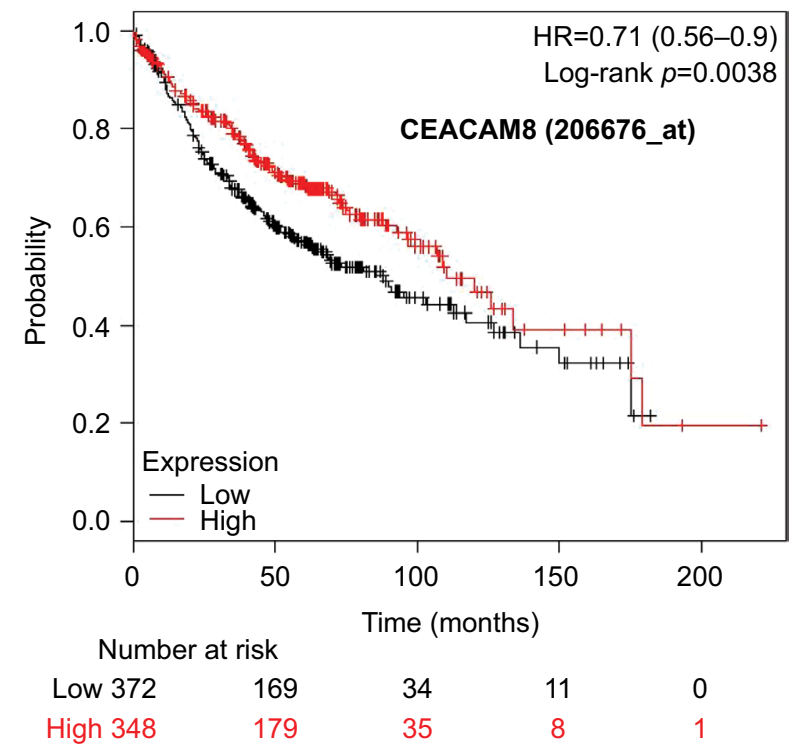

Figure 7 Kaplan-Meier survival curves of the higher and lower expression groups divided by the median value of CEACAM8 (Jetset probe, 206676_at) in patients with adenocarcinoma.

Note: Overall survival was better in the higher expression group $(p=0.0038)$.

Abbreviation: $\mathrm{HR}$, hazard ratio.

with poor OS with a combined hazard ratio of $2.28 .^{41}$ This meta-analysis concluded that preoperative serum CEA levels can predict OS in patients with NSCLC, although high heterogeneity between included studies and publication biases should be taken into consideration.

Little is known about the functions of CEACAMs, particularly impacts on lung cancer tumorigenesis and 


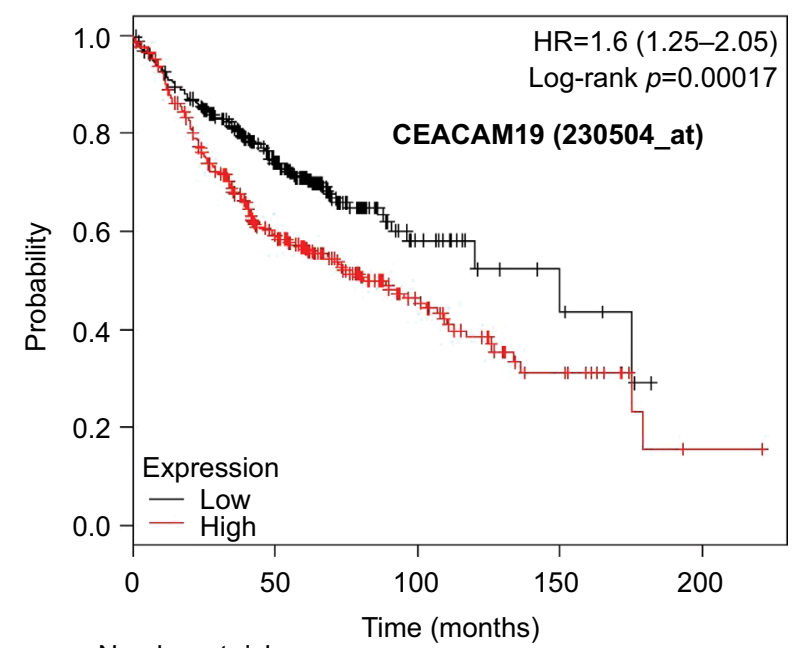

\begin{tabular}{lrlcl}
\multicolumn{5}{c}{ Number at risk } \\
Low 336 & 185 & 23 & 6 & 0 \\
High 337 & 160 & 46 & 13 & 1
\end{tabular}

Figure 8 Kaplan-Meier survival curves of the higher and lower expression groups divided by the median value of CEACAM 9 (Jetset probe, 230504_at) in patients with adenocarcinoma.

Note: Overall survival was worse in the higher expression group ( $p=0.00017)$. Abbreviation: $\mathrm{HR}$, hazard ratio.

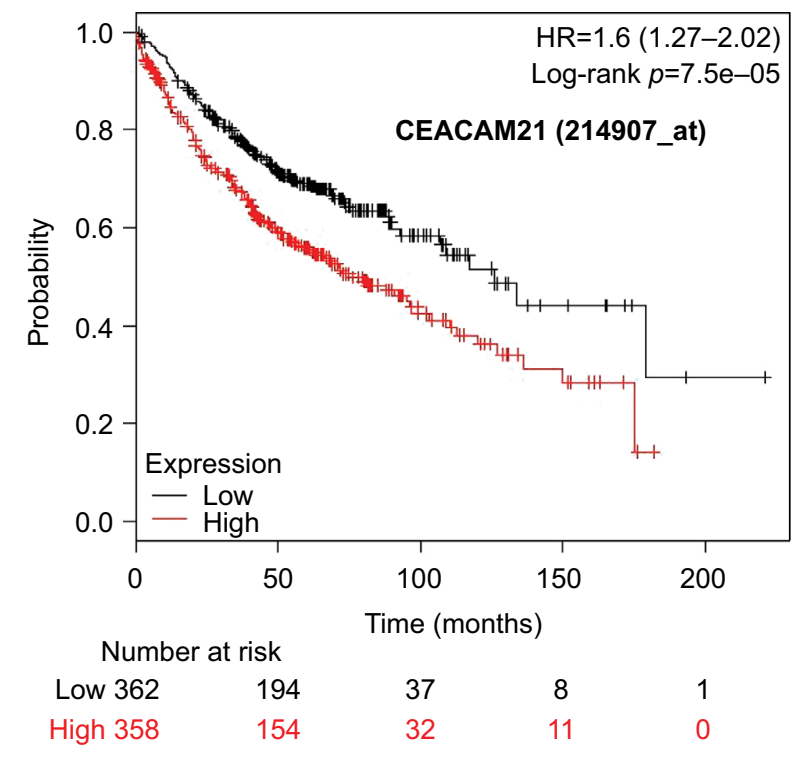

Figure 9 Kaplan-Meier survival curves of the higher and lower expression groups divided by the median value of CEACAM2 I (Jetset probe, 2I4907_at) in patients with adenocarcinoma.

Note: Overall survival was worse in the higher expression group $(p<0.0001)$.

Abbreviation: $\mathrm{HR}$, hazard ratio.

development. However, the functions and prognostic values of CEACAM1, CEACAM5, and CEACAM6 have been investigated in several tumors. For instance, surface expression of CEACAM1-4L in A549 human lung AD cells is reported to play a critical role in differentiation, contactinhibited cell growth, and tumor suppressive functions. ${ }^{42}$ Disturbances in CEACAM1-4L signaling in A549 cells by CEACAM1-4S and other CEACAMs, such as CEACAM5 and CEACAM6, lead to undifferentiated cell growth and malignant transformation. In contrast, multiple clinical studies reported that CEACAM1 overexpression was associated with worse prognosis in melanoma, ${ }^{43}$ gastric cancer, ${ }^{44}$ thyroid cancer, ${ }^{45}$ and NSCLC,${ }^{46}$ suggesting that CEACAM1 contributes to tumor progression. Thus, there are discrepancies concerning the functions of CEACAM1 among these studies. In colorectal cancer, immunohistochemically detected CEACAM6 overexpression in tumor tissues independently predicted poor OS and shortened diseasefree survival, whereas CEACAM1 and CEACAM5 were not significantly related to these outcomes. ${ }^{47}$ In epidermal growth factor receptor mutation-negative lung AD patients, a immunohistochemical study of tumor tissues revealed that CEACAM6 expression was associated with worse prognoses, whereas CEACAM3 expression was associated with better prognoses. ${ }^{48}$ These studies indicated that CEACAM6 overexpression was a worse prognostic factor for selected lung cancer patients.

In the present study, none of the $C E A C A M$ gene family members were predictive of OS in patients with SQ. The most apparent result in this study is that CEACAM expression in lung SQ is not useful to predict OS. In contrast, statistically significant differences in OS were confirmed in NSCLC and AD. Since NSCLC is mainly composed of AD and SQ, differences in OS among NSCLC patients are mostly a reflection of OS differences in $\mathrm{AD}$ patients.

Associations with worse OS in patients with AD were confirmed by higher mRNA expression of CEACAM3, CEACAM4, CEACAM19, and CEACAM21, while CEACAM6 and CEACAM8 were associated with better OS. Since CEACAM6 overexpression is reportedly associated with worse prognosis of various cancers, ${ }^{47-51}$ the results of the present study are unique and should be confirmed in further studies. We found no reports examining serum concentration or expression levels of the other CEACAMs and associated impacts on survival of cancer patients.

For some $C E A C A M$ gene family members, conflicting results were obtained because of the use of unique probes for each gene. This is not surprising because a given gene may be detected by multiple probe sets on an Affymetrix microarray, which can result in inconsistent or even contradictory findings. The cross-reactivity of probes to other genes and multiple transcripts produced by alternative splicing events are plausible reasons. In order to create simple one-to-one mapping between genes and probe sets, a scoring system using a specific algorithm was proposed for Jetset probes as the most reliable, and these probes are identified on the Kaplan-Meier plotter webpage. ${ }^{38}$ The most conflicting results 
were found for the $C E A C A M 1$ probe sets. Because $C E A C A M 1$ has 13 splice variants and each may have a different function in lung cancer progression, ${ }^{42}$ different prognostic significance might be due to the different specificity of the probe set reactive with the different variants. Jetset probe 209498_at for $C E A C A M 1$ indicated better OS. Similarly, conflicting results were found in CEACAM5 and CEACAM7. No differences in OS based on mRNA expression were found for Jetset probe sets 201884_at for CEACAM5 and 206199_at for CEACAM7.

Since there is quite limited information concerning the relationships between overexpressed CEACAMs in tumor tissues and survival of lung cancer patients, data mining using an accessible public database is both reasonable and useful. A major limitation to this study was the limited clinical information of individual patients, thus it was difficult to perform subgroup analyses. However, the total number of included patients was sufficient to obtain reliable results, if interpreted cautiously.

In conclusion, we found statistically significant associations between mRNA expression of CEACAMs and OS of NSCLC patients. These close associations were confirmed in $\mathrm{AD}$, but not in SQ, suggesting that CEACAMs play important roles in progression of AD. Since the impact of expression of individual CEACAMs on OS differed (better, worse, or neutral) based on the gene family members or used probe sets, each CEACAM seems to function through complicated mechanisms. Hence, further studies are necessary to resolve the problems encountered in the present study.

\section{Disclosure}

The authors report no conflicts of interest in this work.

\section{References}

1. Gold P, Freedman SO. Demonstration of tumor-specific antigens in human colonic carcinoma by immunological tolerance and absorption techniques. J Exp Med. 1965;121:439-462.

2. Beauchemin N, Arabzadeh A. Carcinoembryonic antigen-related cell adhesion molecules (CEACAMs) in cancer progression and metastasis. Cancer Metastasis Rev. 2013;32(3-4):643-671.

3. Chevinsky AH. CEA in tumors of other than colorectal origin. Semin Surg Oncol. 1991;7(3):162-166.

4. Hasegawa T, Isobe K, Tsuchiya Y, Oikawa S, Nakazato H, Nakashima I, Shimokata K. Nonspecific crossreacting antigen (NCA) is a major member of the carcinoembryonic antigen (CEA)-related gene family expressed in lung cancer. Br J Cancer. 1993;67(1):58-65.

5. Allard WJ, Neaman IE, Elting JJ, Barnett TR, Yoshimura H, Fritsche HA, Yeung KK. Nonspecific cross-antigen 50/90 is elevated in patients with breast, lung, and colon cancer. Cancer Res. 1994;54(5):1227-1234.

6. Tchoupa AK, Schuhmacher T, Hauck CR. Signaling by epithelial members of the CEACAM family - mucosal docking sites for pathogenic bacteria. Cell Commun Signal. 2014;12:27.

7. Pavlopoulou A, Scorilas A. A comprehensive phylogenetic and structural analysis of the carcinoembryonic antigen (CEA) gene family. Genome Biol Evol. 2014;6(6):1314-1326.
8. Vincent RG, Chu TM, Fergen TB, Ostrander M. Carcinoembryonic antigen in 228 patients with carcinoma of the lung. Cancer. 1975;36(6):2069-2076.

9. Goslin RH, Skarin AT, Zamcheck N. Carcinoembryonic antigen. A useful monitor of therapy of small cell lung cancer. JAMA. 1981;246(19): 2173-2176.

10. Lokich JJ. Plasma CEA levels in small cell lung cancer. Correlation with stage, distribution of metastases, and survival. Cancer. 1982; 50(10):2154-2156.

11. Hsu WH, Huang CS, Hsu HS, Huang WJ, Lee HC, Huang BS, Huang MH. Preoperative serum carcinoembryonic antigen level is a prognostic factor in women with early non-small-cell lung cancer. Ann Thorac Surg. 2007;83(2):419-424.

12. Matsuoka K, Sumitomo S, Nakashima N, Nakajima D, Misaki N. Prognostic value of carcinoembryonic antigen and CYFRA21-1 in patients with pathological stage I non-small cell lung cancer. Eur J Cardiothorac Surg. 2007;32(3):435-439.

13. Matsuguma H, Nakahara R, Igarashi S, et al. Pathologic stage I nonsmall cell lung cancer with high levels of preoperative serum carcinoembryonic antigen: clinicopathologic characteristics and prognosis. J Thorac Cardiovasc Surg. 2008;135(1):44-49.

14. Tomita M, Shimizu T, Hara M, Ayabe T, Onitsuka T. Serum carcinoembryonic antigen level in non-small-cell lung cancer patients with preoperative normal serum level. Gen Thorac Cardiovasc Surg. 2009;57(6):303-306.

15. Nakamura H, Saji H, Marushima H, Kimura H, Koizumi H, Takagi M. Associations between serum carcinoembryonic antigen levels and adenocarcinoma subtypes of the lung. Cancer Treat Commun. 2016; $3: 31-35$

16. Berinstein NL. Carcinoembryonic antigen as a target for therapeutic anticancer vaccines: a review. J Clin Oncol. 2002;20(8):2197-2207.

17. McCann KJ, Mander A, Cazaly A, et al. Targeting carcinoembryonic antigen with DNA vaccination: on-target adverse events link with immunological and clinical outcomes. Clin Cancer Res. 2016;22(19): 4827-4836.

18. Aurisicchio L, Roscilli G, Marra E, Luberto L, Mancini R, La Monica N, Ciliberto G. Superior immunologic and therapeutic efficacy of a xenogeneic genetic cancer vaccine targeting carcinoembryonic human antigen. Hum Gene Ther. 2015;26(6):386-398.

19. Staff C, Magnusson CG, Hojjat-Farsangi M, et al. Induction of IgM, $\operatorname{IgA}$ and IgE antibodies in colorectal cancer patients vaccinated with a recombinant CEA protein. J Clin Immunol. 2012;32(4):855-865.

20. Hong KP, Shin MH, Yoon S, et al. Therapeutic effect of anti CEACAM6 monoclonal antibody against lung adenocarcinoma by enhancing anoikis sensitivity. Biomaterials. 2015;67:32-41.

21. Molina-Pinelo S, Gutierrez G, Pastor MD, et al. MicroRNA-dependent regulation of transcription in non-small cell lung cancer. PLoS One. 2014;9(3):e90524.

22. Duxbury MS, Matros E, Ito H, Zinner MJ, Ashley SW, Whang EE. Systemic siRNA-mediated gene silencing: a new approach to targeted therapy of cancer. Ann. Surg. 2004;240(4):667-674; discussion 675-676.

23. Turriziani M, Fantini M, Benvenuto M, et al. Carcinoembryonic antigen (CEA)-based cancer vaccines: recent patents and antitumor effects from experimental models to clinical trials. Recent Pat Anticancer Drug Discov. 2012;7(3):265-296.

24. Gyorffy B, Surowiak P, Budczies J, Lanczky A. Online survival analysis software to assess the prognostic value of biomarkers using transcriptomic data in non-small-cell lung cancer. PLoS One. 2013;8(12):e82241.

25. You Q, Guo H, Xu D. Distinct prognostic values and potential drug targets of ALDH1 isoenzymes in non-small-cell lung cancer. Drug Des Devel Ther. 2015;9:5087-5097.

26. Raponi M, Zhang Y, Yu J, et al. Gene expression signatures for predicting prognosis of squamous cell and adenocarcinomas of the lung. Cancer Res. 2006;66(15):7466-7472.

27. Zhu CQ, Ding K, Strumpf D, et al. Prognostic and predictive gene signature for adjuvant chemotherapy in resected non-small-cell lung cancer. J Clin Oncol. 2010;28(29):4417-4424. 
28. Hou J, Aerts J, den Hamer B, et al. Gene expression-based classification of non-small cell lung carcinomas and survival prediction. PLoS One. 2010;5(4):e10312.

29. Bild AH, Yao G, Chang JT, et al. Oncogenic pathway signatures in human cancers as a guide to targeted therapies. Nature. 2006;439(7074):353-37.

30. Yamauchi M, Yamaguchi R, Nakata A, et al. Epidermal growth factor receptor tyrosine kinase defines critical prognostic genes of stage I lung adenocarcinoma. PLoS One. 2012;7(9):e43923.

31. Shedden K, Taylor JM, Enkemann SA, et al. Gene expression-based survival prediction in lung adenocarcinoma: a multi-site, blinded validation study. Nat Med. 2008;14(8):822-827.

32. Hammerman PS, Hayes DN, Wilkerson MD, Schultz N, Bose R; Cancer Genome Atlas Research Network. Comprehensive genomic characterization of squamous cell lung cancers. Nature. 2012;489(7417): 519-525.

33. Xie Y, Xiao G, Coombes KR, et al. Robust gene expression signature from formalin-fixed paraffin-embedded samples predicts prognosis of non-small-cell lung cancer patients. Clin Cancer Res. 2011;17(17): 5705-5714.

34. Botling J, Edlund K, Lohr M, et al. Biomarker discovery in non-small cell lung cancer: integrating gene expression profiling, meta-analysis, and tissue microarray validation. Clin Cancer Res. 2013;19(1): 194-204.

35. Rousseaux S, Debernardi A, Jacquiau B, et al. Ectopic activation of germline and placental genes identifies aggressive metastasis-prone lung cancers. Sci Transl Med. 2013;5(186):186ra66.

36. Girard L, Rodriguez-Canales J, Behrens C, et al. An expression signature as an aid to the histologic classification of non-small cell lung cancer. Clin Cancer Res. 2016;22(19):4880-4889.

37. Der SD, Sykes J, Pintilie M, et al. Validation of a histology-independent prognostic gene signature for early-stage, non-small-cell lung cancer including stage IA patients. Thorac Oncol. 2014;9(1):59-64.

38. Li Q, Birkbak NJ, Gyorffy B, Szallasi Z, Eklund AC. Jetset: selecting the optimal microarray probe set to represent a gene. BMC Bioinformatics. 2011;12:474.

39. Grunnet M, Sorensen JB. Carcinoembryonic antigen (CEA) as tumor marker in lung cancer. Lung Cancer. 2012;76(2):138-143.
40. Ford CH, Stokes HJ, Newman CE. Carcinoembryonic antigen and prognosis after radical surgery for lung cancer: immunocytochemical localization and serum levels. Br J Cancer. 1981;44(2):145-153.

41. Wang XB, Li J, Han Y. Prognostic significance of preoperative serum carcinoembryonic antigen in non-small cell lung cancer: a meta-analysis. Tumour Biol. 2014;35(10):10105-10110.

42. Singer BB, Scheffrahn I, Kammerer R, Suttorp N, Ergun S, Slevogt H. Deregulation of the CEACAM expression pattern causes undifferentiated cell growth in human lung adenocarcinoma cells. PLoS One. 2010;5(1):e8747.

43. Thies A, Moll I, Berger J, et al. CEACAM1 expression in cutaneous malignant melanoma predicts the development of metastatic disease. $J$ Clin Oncol. 2002;20(10):2530-2536.

44. Zhou CJ, Liu B, Zhu KX, et al. The different expression of carcinoembryonic antigen-related cell adhesion molecule 1 (CEACAM1) and possible roles in gastric carcinomas. Pathol Res Pract. 2009;205(7):483-489.

45. Liu W, Wei W, Winer D, et al. CEACAM1 impedes thyroid cancer growth but promotes invasiveness: a putative mechanism for early metastases. Oncogene. 2007;26(19):2747-2758.

46. Laack E, Nikbakht H, Peters A, et al. Expression of CEACAM1 in adenocarcinoma of the lung: a factor of independent prognostic significance. J Clin Oncol. 2002;20(21):4279-4284.

47. Jantscheff P, Terracciano L, Lowy A, et al. Expression of CEACAM6 in resectable colorectal cancer: a factor of independent prognostic significance. J Clin Oncol. 2003;21(19):3638-3646.

48. Kobayashi M, Miki Y, Ebina M, et al. Carcinoembryonic antigen-related cell adhesion molecules as surrogate markers for EGFR inhibitor sensitivity in human lung adenocarcinoma. Br JCancer. 2012;107(10):1745-1753.

49. Kim KS, Kim JT, Lee SJ, et al. Overexpression and clinical significance of carcinoembryonic antigen-related cell adhesion molecule 6 in colorectal cancer. Clin Chim Acta. 2013;415:12-19.

50. Chen J, Li Q, An Y, et al. CEACAM6 induces epithelial-mesenchymal transition and mediates invasion and metastasis in pancreatic cancer. Int J Oncol. 2013;43(3):877-885.

51. Zang M, Zhang B, Zhang Y, et al. CEACAM6 promotes gastric cancer invasion and metastasis by inducing epithelial-mesenchymal transition via PI3K/AKT signaling pathway. PLoS One. 2014;9(11):e112908.
Current Biomarker Findings

\section{Publish your work in this journal}

Current Biomarker Findings is an international, peer-reviewed, open access journal publishing original research, reports, reviews and commentaries on all areas of biomarker research. The manuscript management system is completely online and includes a very quick and fair peer-review system. Visit http://www.dovepress.com/testimonials.php to read real quotes from published authors.

\section{Dovepress}

\author{
m/testimonials.php
}

